\title{
Plein air et jeux de plein air dans les écoles primaires de Metz (1919-1932)
}

\section{Jogos ao ar livre nas escolas primárias de Metz (1919-1932)}

\section{Metz primary schools outdoors games (1919-1932)}

Laurent Grün*

\begin{abstract}
RÉSUMÉ
Les jeux de plein air scolaires organisés par la ville de Metz entre 1919 et 1932 représentent une expérience originale. Créés sous le régime du Reichsland en 1903, ils se poursuivent cependant après la première guerre mondiale, après que Metz soit revenue à la France. Organisés par des instituteurs volontaires, ils proposent aux élèves qui le désirent des activités physiques ou sportives en dehors des heures scolaires. L'ambition est de développer la santé des élèves dans un cadre moins contraignant que celui de la gymnastique obligatoire de l'école, tout en les éloignant des tentations de la rue. Ces jeux de plein air s'adressent sans distinction de sexe aux garçons comme aux filles et sans distinction confessionnelle aux catholiques, aux protestants, aux laïcs. Pour ce faire, divers lieux d'activité sont investis, toujours en plein air. Les activités choisies relèvent le plus souvent des jeux traditionnels, mais peuvent aussi être de la culture physique, des activités sportives, des promenades, des bains de plein air. De 1919 à 1932, plusieurs centaines d'écolières et d'écoliers messins ont donc bénéficié d'une éducation corporelle morale et hygiénique. Celle-ci s'est déroulé dans un cadre moins strict que celui de l'école, sous la direction d'instituteurs qui utilisaient des pédagogies plus actives et moins traditionnelles qu'à l'école.
\end{abstract}

Mots clés : Extérieur. Jeux. Éducation physique. Étudiant. Metz.

* Université de Lorraine. Nancy, France. E-mail: laurent.grun@univ-lorraine.fr - https:// orcid.org/0000-0002-9682-1692 


\title{
RESUMO
}

Os jogos escolares ao ar livre organizados pela cidade de Metz entre 1919 e 1932 representam uma experiência original. Criados sob o regime do Reichsland em 1903, eles continuaram presentes mesmo após a Primeira Guerra Mundial, depois que Metz voltou a ser parte do território francês. Organizados por professores primários voluntários, atividades físicas ou esportivas eram ofertadas aos alunos, fora do horário escolar. A ambição era desenvolver a saúde dos estudantes num quadro menos restritivo do que o proporcionado pela ginástica escolar obrigatória, mantendo-os com isso afastados das tentações da rua. Nesses jogos ao ar livre não havia uma distinção de sexo e nem uma diferenciação confessional (católicos, protestantes e laicos). Para isso, foram criados vários locais de atividade, sempre ao ar livre. As ações escolhidas eram na maioria das vezes os jogos tradicionais, mas também podiam ser cultura física, atividades esportivas, passeios, banhos ao ar livre. De 1919 a 1932, várias centenas de alunos e alunas de Metz se beneficiaram de uma educação física moral e higiênica. Isso ocorreu em um quadro menos estrito do que o existente no interior da escola, sob a direção de professores primários que utilizavam de pedagogias mais ativas e menos tradicionais do que as praticadas no contexto escolar.

Palavras-chave: Jogos ao ar livre. Educação Física. Aluno. Metz.

\begin{abstract}
The city of Metz organized outdoor games for students between 1919 and 1932. This should be considered as an innovative experiment. Created under the Reichsland's regime in 1903, this organization did not stop its activities after World War 1, when Metz returned to the French homeland in 1918. Volunteer teachers kept on proposing sporting or physical activities to young students, aged 6-13, who were willing to participate outside classtime. The primary goal aimed to promote schoolchildren health in a less strict educational environment and at the same time to keep them away from streets temptations. Each sex could take part into these outdoor games and no one could be excluded, based on his or her religious affiliation. Activities took place in various venues, but always outdoor. They were often traditional games or child's plays, but sometimes pupils were teached physical education or sports. Some of them were brought to walks or river baths. From 1919 to 1932, several hundreds of schoolgirls and schoolboys from Metz benefited from these specific forms of physical education, aiming both sanitary and moral improvement. Outdoor environment was less rigorous than compulsory school and teachers could use more active teaching and less traditional methods.
\end{abstract}

Keywords: Outdoor Games. Physical education. Student. Metz. 


\section{Introduction}

Dans l'entre-deux-guerres, la municipalité de Metz permet à un groupe d'instituteurs volontaires de faire pratiquer une éducation physique facultative aux élèves qui y consentent. Cette politique volontariste d'encadrement de la jeunesse, mise en œuvre dès 1903, est consolidée dans l'entre-deux-guerres, davantage à l'instigation des enseignants qu'à celle de la municipalité. La ville de Metz, qui y trouve cependant un intérêt certain, accepte de subventionner cette organisation de 1919 à 1932. Le but est de permettre aux élèves messins des écoles primaires de bénéficier d'une éducation corporelle destinée à compléter l'éducation physique dispensée lors de l'enseignement scolaire obligatoire, grâce à l'emploi de jeux pratiqués en plein air. L'ambition avouée est bien de permettre aux jeunes messins et messines d'acquérir une meilleure santé. Elle se double de préceptes moraux avoués: soustraire ces enfants et adolescents à l'oisiveté ou aux tentations de la rue, éviter de les laisser livrés à eux-mêmes lors de leur temps libre.

Il s'agira dans un premier temps de détailler les buts de ces jeux de plein air et de montrer que l'éducation corporelle visée pour les élèves messins revêt des aspects d'ordre physique, mais également moral. Dans un second temps, nous constaterons qu'entre 1919 et 1932, les jeux de plein air, au départ dévolus aux deux sexes, finissent progressivement par devenir l'apanage des garçons. Par contre, ils s'adressent sans restriction à des élèves de différentes confessions, catholiques, protestants et même aux laïcs. Enfin, nous nous attacherons à la déclinaison de ces jeux : le plein air, tel qu'il est entendu par les responsables municipaux aussi bien que par les instituteurs messins, reste une notion souple (SURREL, 1947). En effet, les activités peuvent se dérouler aussi bien dans des lieux bien différents les uns des autres. Les jeux en eux-mêmes sont variés, puisqu'ils peuvent aussi bien consister en un jeu traditionnel qu'en une partie de football. De ce fait, ils octroient aux garçons et filles qui les fréquentent une éducation corporelle variée et souvent basée sur des procédés moins rigides que ceux observés dans le cadre de la classe.

L'originalité de cette organisation des jeux de plein air tient à plusieurs aspects : tout d'abord, elle naît dans une région placée sous l'autorité allemande depuis 1871. Mais malgré le changement de régime et le retour à la France en 1918, les jeux de plein air se pérennisent en dépit des particularismes locaux. Ils se déroulent dans un espace singulier, le périscolaire, ni intégré à l'école, ni à sa marge, qui leur octroie une relative liberté pédagogique dans leur fonctionnement. Enfin, ils semblent dépasser les antagonismes confessionnels, ce qui est peu courant dans l'entre-deux-guerres, aussi bien dans le cadre scolaire que dans le cadre associatif. 


\section{Méthodologie}

L'étude des archives municipales de Metz (1 R 270) constitue notre source principale. Ce dossier $1 \mathrm{R} 270$ est constitué de courriers de la mairie vers les enseignants qui encadrent le plein air, et vice-versa. Il contient également des rapports annuels d'activité qui détaillent où sont scolarisés les d'enfants concernés dans les différentes écoles messines, qui les encadre et parfois quelles sont les activités précisément dispensées à ces jeunes messines et messins. Malheureusement, certaines années scolaires de la période 1919-1932 ne font l'objet d'aucun document archivé. Cependant, ceux qui sont mis à notre disposition sont suffisamment riches pour permettre de proposer une lecture réaliste de pratiques de plein air et de leur intérêt dans le cadre d'une éducation corporelle spécifique des élèves volontaires de la ville de Metz.

\section{Mise en perspective historique}

Metz est une ville française jusqu'en 1871. Mais suite à la défaite contre la Prusse, la France cède l'Alsace et la Moselle à l'Allemagne, en application du traité de Francfort signé le 10 mai 1871. Metz, qui était le chef-lieu de la Moselle, appartient donc dorénavant au Reichsland, constitué par ces provinces d'Alsace et de Moselle jusqu'en 1918. Elle redevient officiellement française à partir du traité de Versailles signé le 28 juin 1919 (ROTH, 1976). C'est donc sous le régime allemand que les activités et jeux de plein air sont mises en place dans les écoles de Metz dès 1903 (CHARPIER, 1996). A cette période se forment en Moselle des institutions qui, comme en Alsace, soit participent à la lutte contre la germanisation, soit à l'inverse tentent de renforcer le processus d'assimilation au Reich allemand ${ }^{1}$. Mais après 1918, l'organisation des jeux de plein air de la ville de Metz dépend dorénavant du régime français. Il est vraisemblable qu'avant 1918 à Metz, les jeux ont été encadrés par des instituteurs d'origine française, mais qui avaient une maîtrise parfaite de la langue allemande, puisque les enseignements à l'école se font en allemand dès 1873. Mais dès 1918, après le passage au statut français, c'est le français qui redevient la langue obligatoire en Alsace-Moselle. Plusieurs instituteurs conservent dans 
l'animation de ces jeux de plein air des prérogatives qu'ils remplissaient déjà dans le Reichsland, ce qui pourrait laisse penser qu'ils n'utilisaient pas cet espace de fonctionnement pour véhiculer des idées germanophiles. Nous ne bénéficions pas des dossiers de carrière de ces enseignants, ni de celles et ceux qui viendront compléter l'effectif des volontaires pour l'encadrement du plein air. Mais ces derniers sont forcément des français d'origine, puisqu'en 1918 les instituteurs allemands ont été forcés de quitter leur poste en Moselle (QUIRIN, 2014). Mais en réalité, le plein air et les jeux de plein air des écoles de Metz sont des activités périscolaires, et en ce sens elles semblent s'émanciper à la fois de certaines obligations confessionnelles de l'enseignement scolaire obligatoire, mais également de potentielles convictions nationalistes.

\section{Les objectifs des jeux de plein air et les moyens octroyés}

Dans un rapport envoyé au conseil municipal de la ville de Metz (ARCHIVES MUNICIPALES DE METZ, 1922), le directeur de l'école SaintMaximin, Mr François mentionne que ces jeux, créés par un comité en 1902, fonctionnent depuis l'année 1903. Malheureusement, aucune précision n'est apportée quant à la constitution de ce comité. Émane-t-il de parents d'élèves, de membres d'une ligue d'enseignement, d'une association pour l'encadrement de la jeunesse ? Les archives n'apportent pas de réponse. Mais il est plus que vraisemblable que le contexte social et politique, porté par le souci de l'hygiène, a influencé les membres de ce comité responsable de la création des jeux de plein air à Metz, qu'ils aient été francophiles ou germanophiles.

Du côté allemand, la création du Deutsche Jugendbewegung (Mouvement de la Jeunesse allemande) en 1896, affecte les mentalités en termes d'éducation des corps par le recours à l'espace naturel (FUCHS ; STUMPP, 2013). Et si par contre les membres de ce comité avaient une inclination à la francophilie, ils ne pouvaient pas ignorer cette volonté d'encadrer la jeunesse en dehors du temps scolaire par la troisième république en France. Ce désir prend des formes diverses : patronages, mouvements de jeunesse, qui désirent œuvrer pour faire de l'enfant un futur membre de la société, gardien des valeurs catholiques ou républicaines (LE BON, 2003). Mais si nous ignorons si les membres du comité qui ont institué les jeux de plein air à Metz étaient francophiles ou germanophiles, on sait en revanche que $\mathrm{Mr}$ Rech, directeur de l'école de la Monnaie, coordonne jusqu'en 1918 un groupes d'enseignants des écoles primaires chargés de dispenser ces jeux et exercices (ARCHIVES MUNICIPALES DE METZ, 
1920a). Mais suite à son départ en retraite qui occasionne une désorganisation (ARCHIVES MUNICIPALES DE METZ, 1920a), le directeur de l'école SaintMaximin, Mr François, demande aux élus messins de pouvoir réorganiser ces cours et d'en assurer la coordination entre les différentes écoles primaires de la ville de Metz. Il met en avant son expérience, puisqu'il faisait partie des enseignants chargés de de l'encadrement du plein air depuis sa première année de fonctionnement en 1903. Le premier but avoué de ces jeux de plein air hors temps scolaire est éminemment hygiénique : " ces cours qui sont d'une grande importance pour l'éducation et le développement physique des élèves » (ARCHIVES MUNICIPALES DE METZ, 1920a). Il est possible qu'à l'origine ce comité périscolaire ait été influencé par le Turnen enseigné dans les écoles du Reichsland, une gymnastique d'inspiration pangermaniste qui réaliserait une synthèse idéale entre santé corporelle et énergie intellectuelle (DREIDEMY, 2008). Il est probable également que les discours hygiénistes des médecins, que ce soit en France ou en Allemagne, ont pesé d'un poids réel sur cette proposition de jeux de plein air. Ainsi, l'Association allemande pour l'hygiène scolaire, tout comme en France la Ligue des Médecins et des Familles se sont donnés comme but de ménager à l'école la santé des enfants, en militant pour que l'on donne « à la vie au grand air toute l'importance » dès l'âge scolaire (CHABOT, 1903, p. 547).

Des deux côtés de la frontière, l'intention est bien de doter les enfants scolarisés d'espace, d'air, de lumière, afin de lutter contre l'anémie, le surmenage scolaire, la fatigue, voire les maladies contagieuses. Il s'agit donc de faire acquérir la santé, afin, pour les francophiles, de lutter contre une prétendue dégénérescence de la race française (VIGARELLO, 1999), de rendre les corps sains. Côté allemand, des appels pour une réforme scolaire relaient un vaste mouvement hygiéniste qui se constitue à partir du milieu du XIX ${ }^{\mathrm{e}}$ siècle par la création d'associations de dimension nationale, telles que le « Zentralverein für Körperpflege in Volk und Schule » (Association nationale pour la culture physique dans et hors de l'école) en 1882 (PERNY, 2014). En Alsace-Moselle, le gouverneur du Reichsland von Manteufel crée en 1882 une commission pour l'amélioration de la santé des enfants et du fonctionnement de l'école, qui recommande notamment l'usage des jeux de plein air. Elle est suivie en 1891 par la création du Zentral-Ausschuss zur Förderung der Jugend-und Volksspiele in Deutschland (Comité national pour la promotion des jeux scolaires et des jeux populaires allemands) (PERNY, 2014). Nul doute que ce contexte social influence la création de ces jeux de plein air à Metz en 1902. Cependant, la justification du plein air à l'école ou aux portes des écoles de Metz ne saurait être exclusivement corporelle. Elle se double d'une argumentation morale depuis 1903 : « Déjà à cette époque on avait envisagé que c'était un moyen, et pour 
ainsi dire le seul, d'enlever les enfants de la rue en dehors des heures de classe, et de leur donner l'occasion de se livrer à des jeux et exercices fortifiants en plein air » (ARCHIVES MUNICIPALES DE METZ, 1922). L'encadrement de la jeunesse dans un but d'éducation civique fournit donc un autre prétexte primordial pour l'instauration de ces jeux. Il s'agit d'empêcher les enfants de s'adonner à de mauvaises habitudes, telles que l'oisiveté, la petite délinquance ou l'alcoolisme.

De manière concomitante, fin XIXème et début XXème, plusieurs associations voient le jour en France pour propager une nouvelle entreprise hygiéniste, afin de lutter contre la dépopulation, la syphilis, la tuberculose, l'alcoolisme (ROSANVALLON, 1990), comme l'Alliance d'hygiène sociale créée en 1905 et présidée par le radical Léon Bourgeois. Quelle que soit l'inclination des membres du comité fondateur de ces jeux de plein air, germanophile, francophile, voire neutre, ces derniers n'ont pu échapper aux influences d'un mouvement hygiénique qui se répand des deux côtes du Reichsland, mais également en son sein. Lorsque la première guerre mondiale prend fin et que la Moselle redevient française, ce souci ne s'estompe pas. Au contraire, la volonté de régénérescence de la race (BANCEL ; GAYMAN, 2002), présente en France depuis les années 1850 mais surtout après 1871 se renforce. Il s'agit de revitaliser les corps de toute une population, enfants compris, meurtris par la guerre de 1914-1918. Pour cela, la gymnastique à l'école revêt une importance de premier ordre dans l'esprit des médecins et des hygiénistes (SAINT MARTIN, 1998). Mais le gouvernement, ou à défaut des personnalités politiques ou industrielles, tentent d'assurer la continuité de l'éducation physique en dehors de l'école, en favorisant le développement les mouvements de jeunesse, tels que le scoutisme (GUÉRIN, 1981), les patronages les œuvres d'assistance. C'est bien le cas dans cet exemple de l'encadrement de la jeunesse messine : «La nécessité d'enlever les enfants de la rue en dehors des heures de classe, et de développer leurs forces et leur agilité existe encore à un plus haut degré aujourd'hui que lors de la création de l'organisation » (ARCHIVES MUNICIPALES DE METZ, 1922). Comme dans toute la France, la Moselle de l'immédiat après-guerre ressent le besoin d'encadrer les enfants, parfois au moyen d'activités physiques et sportives dans les patronages, aussi bien laïcs que confessionnels. Cette tendance bien affirmée depuis la fin du XIXème siècle (CRUBELIER, 1979), se confirme après 1918.

Les dispositions prises par la ville de Metz, qui décident du maintien des jeux de plein air en 1919, anticipent celles prises par le gouvernement français : avec la parution des Instructions officielles de 1923 (IO 1923), qui font office de programme scolaire pour l'enseignement primaire, l'accent est porté sur le plein air. Dans cette conjoncture d'après-guerre, rappelons-le, l'ambition de régénérer 
les corps demeure première pour les gouvernants. Ainsi le député de Paris Henri Paté interpelle-t-il le Parlement au début des années 1920 sur la nécessité de développer dès la première jeunesse des habitudes d'hygiène indissociables d'une éducation morale (THIBAULT, 1995). Dans ce cadre, les invitations au plein air renvoient certes à l'hygiène, mais également à l'action salutaire des expériences vécues par la jeunesse (RAUCH, 1983). C'est la position que défend le coordonnateur de ces jeux à Metz, lorsqu'il affirme que pour les 5 groupes de garçons et les 2 groupes de filles qui ont fonctionné en 1924, " l'organisation des jeux poursuit un but vraiment humanitaire » (ARCHIVES MUNICIPALES DE METZ, 1924). La volonté d'arracher les élèves à des tentations douteuses ou immorales reste une constante qui se perpétue durant toute l'existence de l'organisation. A la veille de la disparition des jeux en 1932, le directeur François dresse un bilan plus que satisfaisant pour l'année 1931. Selon lui, les élèves des 8 groupes de garçons et de l'unique école filles de représentée, ont retiré de leur assiduité des effets éminemment bénéfiques. Pendant les 25 séances du jeudi après-midi, les quelques 40 à 50 élèves de chaque groupe, «pendant le temps qu'ils passaient sur les places de jeu, étaient écartés de la rue et fortifiaient leur santé par de bons exercices » (ARCHIVES MUNICIPALES DE METZ, 1932a). Ainsi, tout au long de la période 1919-1932, le souci hygiénique s'est toujours marié avec le principe de moralisation chez les enseignants responsables de ces élèves.

\section{Régénérer la jeunesse scolaire messine : quels enseignants, pour quels élèves ?}

Dans le Reichsland, le régime scolaire est spécifique : ce ne sont pas les lois Ferry qui sont appliquées, comme en France, mais les lois Guizot. D'autre part, l'enseignement se réalise en allemand et dans le cadre de l'école obligatoire, les écoliers sont effectivement soumis aux exercices issus du Turnen. Mais dès lors qu'il s'agit d'exercices et de jeux dans le cadre des horaires non obligatoires, l'éventail de choix des activités est plus large. Il est probable qu'entre 1903 et 1918, les élèves des écoles de Metz ont pratiqué des jeux de balle qui étaient au programme des activités dont Grenzball, Tamburinball, Faustball, Balljagd, Fussball... Schlaghölzer ${ }^{2} .$. A partir du rattachement de la Moselle à la France,

2 Allgemein Unterrichtende für Einführung in die Jugend, 1892. On peut approximativement traduire ces jeux respectivement par « ballon prisonnier, balle au tambourin, balle au poing, ballon chasseur, football, caverne. 
les instituteurs et institutrices ont dû s'inspirer du Manuel d'exercices physiques et de jeux scolaires de 1908, qui constitue le programme obligatoire d'éducation physique en France. Ce dernier stipule : « La leçon doit se donner, autant que possible, dans la cour, à l'air libre » (MANUEL..., 1908). De fait, comme leur intitulé l'indique, les jeux de plein air facultatifs des écoles de Metz se déroulent également à l'extérieur.

Malheureusement, nous n'avons pas de traces précises de l'identité des élèves qui fréquentent ces jeux de plein air. Nous savons que les élèves concernés ont pour la plupart entre 6 et 13 ans. Nous connaissons les effectifs par année scolaire, ainsi que la répartition des élèves par sexe et parfois par âge. Mais nous n'avons pas des listes nominatives, ni d'indication particulière quant à la catégorie socio-professionnelle de leurs parents. Nous pouvons émettre l'hypothèse que ces jeunes filles et jeunes garçons appartiennent à des familles pour lesquelles la religion et la pratique d'un culte ne revêtent qu'une importance mineure. En effet, pour les garçons notamment, l'organisation des jeux de plein air est concurrencée par « des organisations analogues dans les cercles paroissiaux » (ARCHIVES MUNICIPALES DE METZ, 1923). Cette offre, qui peut par exemple se concrétiser dans le scoutisme (FUCKS, 2013), s'inscrit dans une mouvance qui n'est pas nouvelle, mais qui retrouve une vitalité nouvelle après la guerre. Cet éventail de potentialités extra-scolaires, souvent confessionnelles, amène les élus messins à se questionner sur l'opportunité du maintien de ces jeux pour l'exercice 1923. Bien que nous n'ayons pas connaissance du procès-verbal des délibérations, il est possible que la volonté de proposer un encadrement qui soit laïc, donc conforme aux idéaux de la IIIème République, ait orienté favorablement les délibérations des membres du conseil municipal de Metz. Malgré le statut particulier de l'Alsace-Moselle en matière de droit confessionnel, on ne peut exclure l'hypothèse que certains des instituteurs et des parents à l'initiative de ces jeux aient été des laïcs. On s'aperçoit en effet que ces jeux sont proposés aussi bien aux garçons qu'aux filles, aux élèves qui fréquentent une école protestante comme à ceux qui fréquentent une école catholique ou laïque. Cette action s'inscrit aussi sans doute dans la perspective d'aider les élèves les moins favorisés socialement, ceux qui fréquentent l'école du peuple et qui ne resteront sur les bancs de l'école que jusqu'à l'âge de 13 ans, à l'inverse de leurs condisciples de l'école des notables (PROST, 1968).

A partir de 1919, la régénération morale et physique de la jeunesse scolaire messine adopte des formes diverses qui ont anticipé les directives ministérielles. En effet, les IO 1923 à destination de l'enseignement primaire, insistent sur trois urgences : «Plus d'air, plus d'aisance plus de liberté » (IO, 1923, p. 7). Il est vrai qu'après 1920 en France, les pratiques de plein air connaissent un développement conséquent, comme pourrait en témoigner le développement de l'hébertisme 
(BANCEL; GAYMAN, 2002). Le texte des IO 1923 proclame la nécessité de leçons d'éducation physique en plein air et recommande de «saisir toutes les occasions pour utiliser les espaces libres qui peuvent être mis à la disposition de l'école» (IO, 1923, p. 2). Il est établi de surcroît que les installations disponibles en France sont dans de nombreux cas soit défectueuses, soit en nombre insuffisant pour beaucoup d'écoles (GAY-LESCOT, 1999). En l'occurrence, recourir à la pratique du plein air est également un moyen de pallier un déficit d'équipements de sport ou dédiés au développement corporel qui auraient pu ou dû être dévolus à la pratique des différentes formes de gymnastique scolaire.

Lorsque Mr François, le directeur de l'école Saint-Maximin, demande à être chargé de la direction de ces jeux en remplacement de son prédécesseur, il précise que pour l'année 1918-1919, ces activités n'ont concerné que 2 groupes de garçons (chacun comprenant 40 élèves), le sien et celui de l'instituteur de l'école protestante de la Grève (ARCHIVES MUNICIPALES DE METZ, 1920b), Mr Wehrung. Cependant, pour l'été $1921^{3}$ est organisé un fonctionnement pour 5 écoles de garçons (dont 2 protestantes), 7 écoles de filles (mais en réalité 9 groupes) dont une protestante. La moitié de ces groupes de filles est encadrée par des religieuses, ce qui n'est guère étonnant puisqu'en raison du particularisme local, la Moselle compte en 1920 encore 45\% d'institutrices congréganistes contre 55\% d'institutrices laïques (KIEFFER, 1994). Les séances de jeux de plein air se déroulent à partir de Pâques : les garçons bénéficient selon les écoles de 25 à 30 séances de deux heures, alors que les filles n'ont droit qu'à 15 séances. Le décompte des années 1921 et 1922 fait apparaître une fréquentation moyenne de plus de 36 garçons présents par groupe et par séance et de 31 filles. Au fil des années, entre 1924 et 1932, le directeur François enregistre des fluctuations relatives au fonctionnement des groupes. En 1924 et 1925, plusieurs groupes n'ont pu fonctionner, «l'un faute d'agrès nécessaires pour les jeux, les quatre autres faute de surveillants » (ARCHIVES MUNICIPALES DE METZ, 1924). Il est sans doute possible de questionner le taux de rémunération des institutrices et instituteurs qui dirigent les séances de jeu. La rémunération effective est de 6, 25 francs pour une séance de deux heures en 1922 et passe à 8 francs pour deux heures en 1922. Elle reste modeste au regard d'autres indemnités parfois perçues par les instituteurs, lorsqu'ils occupent les fonctions de secrétaire de mairie ou d'organiste à l'église (KIEFFER, 1994).

En revanche, l'absence d'agrès semble être un prétexte discutable, dans la mesure où justement, il n'existe pas d'imposition spécifique quant à la nature des activités ni quant au lieu de pratique. En 1925 encore, ce sont 3 groupes sur

3 Les archives ne contiennent aucune référence à l'année 1920. 
les 11 prévus qui ne fonctionnent pas, pour cause de maladie des surveillants (ARCHIVES MUNICIPALES DE METZ, 1925). Le nombre de 25 séances délivrées aux filles, devenu identique à celui des garçons, démontre malgré tout une volonté des enseignants de ne pas créer des inégalités de traitement entre les deux sexes. Par contre, en 1924 et 1925, la baisse des effectifs féminins est indéniable. Seuls 2 groupes encadrés par 2 religieuses subsistent sur les 9 décomptés en 1921, alors que dans le même temps, les effectifs garçons restent stables de 1921 à 1925. Les jeunes filles ou leurs familles sont-elles de moins en moins attirées par ces pratiques périscolaires ? La diminution de la fréquentation féminine est-elle due à une défection du personnel enseignant ? La rétribution insuffisante aux yeux des enseignants, le supplément de travail en dehors du temps scolaire, les problèmes liés à une éventuelle lassitude, la recherche de nouveaux lieux de pratique, de surcroît non abrités... peuvent constituer des motifs d'abandon pour les institutrices et instituteurs. Après 1926, le décompte des effectifs est rendu impossible, car on ne retrouve plus de trace de courrier du directeur François dans les archives jusqu'en 1932, date de son tout dernier rapport annuel (ARCHIVES MUNICIPALES DE METZ, 1932a). Il s'agit d'un compte-rendu de l'activité des groupes lors de l'année scolaire 1931. Les archives qui concernent cette année sont particulièrement intéressantes, car la mairie a invité chaque enseignante et enseignant en charge d'un groupe à fournir un rapport détaillé d'activité pour compléter le compte-rendu global de coordination du directeur François (ARCHIVES MUNICIPALES DE METZ, 1932c). L'analyse de ces rapports individuels témoigne d'un accroissement de l'écart de fréquentation entre garçons et filles, puisque 8 groupes de garçons ont fonctionné alors qu'un seul groupe de filles a été encadré, celui de l'école Saint-Maximin par la religieuse congréganiste sœur Karst.

TABLEAU DE FREQUENTATION DES JEUX ENTRE 1918 ET 1931

\begin{tabular}{ccccccc}
\hline Année & $\begin{array}{c}\text { Groupes } \\
\text { G* }\end{array}$ & $\begin{array}{c}\text { Moyenne } \\
\text { effectif G }\end{array}$ & $\begin{array}{c}\text { Nombre } \\
\text { séances G }\end{array}$ & $\begin{array}{c}\text { Groupes } \\
\mathbf{F}^{*}\end{array}$ & $\begin{array}{c}\text { Moyenne } \\
\text { effectif F }\end{array}$ & $\begin{array}{c}\text { Nombre } \\
\text { séances F }\end{array}$ \\
\hline $\mathbf{1 9 1 8 - 1 9 1 9}$ & 2 & 40 & 33 & 0 & 0 & 0 \\
$\mathbf{1 9 2 1}$ & 5 & 37,6 & & 9 & 33 & \\
$\mathbf{1 9 2 2}$ & 4 & & 19 & 6 & & 11 \\
$\mathbf{1 9 2 3}$ & 7 & & 20 & 7 & & 17 \\
$\mathbf{1 9 2 4}$ & 5 & & 24 & 2 & & 22 \\
$\mathbf{1 9 2 5}$ & 6 & & 24 & 2 & & 25 \\
$\mathbf{1 9 3 1}$ & 8 & 45 & 25 & 1 & De 5 à 50 & 25 \\
\hline
\end{tabular}

*Chaque groupe étant encadré par une ou un enseignant(e), le nombre de groupe équivaut au nombre d'enseignant(e)s.

* $\mathrm{G}=$ garçons $; \mathrm{F}=$ filles.

SOURCES: Archives Municipales de Metz. 
Les données enregistrées grâce aux différents rapports annuels du directeur François inclinent à différentes constatations. Tout d'abord, l'écart de fréquentation s'est accru entre garçons et filles. Alors que dans l'immédiat après-guerre, le taux d'encadrement des filles est supérieur à celui des garçons, il diminue pour s'effondrer en 1931. En réalité, l'effondrement a été brutal entre 1923 et 1924, puisque le nombre de groupes de filles est passé de 7 à 2 . Dans le même temps, le taux d'encadrement des garçons a connu peu de fluctuations et augmente même en 1931. En sus des hypothèses déjà émises, on peut questionner les représentations liées à la supposée fragilité féminine en vigueur dans la société française (ZANCARINI-FOURNEL, 2005) et dans le monde de l'éducation physique en particulier (OTTOGALLI-MAZZACAVALLO ; LIOTARD, 2012). Et l'endurcissement au plein air, voire au grand air, correspond davantage aux représentations d'un corps viril, donc masculin (ARNAUD, 1996). Pourtant, pour les groupes de filles qui bénéficient de cet encadrement, le nombre de séances annuelles a progressivement augmenté pour devenir équivalent à celui des garçons. D'autre part, il apparait que si l'effectif moyen d'un groupe peut varier, le plus souvent c'est d'une quarantaine d'élèves dont les enseignants ont à s'occuper. Ces chiffres ne sont pas anormaux, dans la mesure où pour l'année 1931-1932, on compte pour la ville de Metz un instituteur pour 39 élèves (KIEFFER, 1994). De surcroît, les rapports d'activité individuels des enseignants soulignent que parfois en 1931, ils peuvent s'occuper d'élèves de plusieurs niveaux différents. Ainsi, l'instituteur Rohr de l'école Saint-Eucaire dirige-t-il quatre niveaux de classe $\left(6^{\text {ème }}, 5^{\text {ème }}, 4^{\text {ème }}\right.$ et $2^{\text {ème }}$ niveaux $)$. Il est probable que les jeux proposés aux élèves sont identiques quel que soit leur âge, l'instituteur ayant la possibilité de doser l'intensité ou la difficulté des exercices en fonction leur nature ainsi que de l'âge des différents publics présents.

\section{Régénérer la jeunesse scolaire messine : quelles activités proposer ?}

Les lieux d'exercices des jeux de plein air de la ville de Metz sont variés, dans la mesure où les enseignants et leurs élèves quittent le cadre de la salle de classe. Pour des raisons à la fois pratiques et idéologiques, les endroits choisis pour les jeux et exercices correspondent à « toute pratique réalisée en plein air, c'est-à-dire en dehors de toute enceinte fermée » (SAINT-MARTIN, 2014, p. 23). Il s'agit, d'exposer les enfants au milieu naturel, aux bains de soleil, au grand air, avec des visées hygiénistes (VILLARET ; SAINT-MARTIN, 2004). A Metz, parfois, ce sont les lieux publics qui sont investis, tels que la place 
d'exercices de Chambières, la place de l'hôpital militaire à Plantières, la place près de la station de TSF à Queuleu (ARCHIVES MUNICIPALES DE METZ, 1932a). Il peut également s'agir d'installations sportives municipales, comme le stade de Bellecroix ou le terrain de sport de l'île du Saulcy ${ }^{4}$, fréquentés parfois par le groupe de filles de sœur Karst ou celui des garçons de l'instituteur Bonnet (ARCHIVES MUNICIPALES DE METZ, 1932b). Certains enseignants se rendent des secteurs boisés, comme l'ancienne enceinte militaire du fort de Queuleu. En réalité, la proximité géographique avec l'école est un critère significatif de choix dans la plupart des cas. Lorsque les intempéries rendent le terrain impraticable, comme c'est le cas pour la place militaire de Chambières, l'enseignant se rabat tout de même sur la cour de son école (ARCHIVES MUNICIPALES DE METZ, 1932g).

Mais quelles sont les pratiques corporelles effectivement dispensées aux élèves des écoles de Metz ? Le directeur François, coordonnateur des jeux de plein air, distingue bien les activités proposées de celles qui sont prévues dans les les IO 1923, qui contiennent de la gymnastique à base d'exercices respiratoires, d'assouplissements et de la gymnastique d'application telle qu'en propose la méthode naturelle de Georges Hébert (DELAPLACE, 2000). Dans l'agglomération messine, ces jeux de plein air sont donc bien complémentaires de l'éducation physique obligatoire : " Ce ne sont pas des séances de gymnastique, ce sont des séances qui sont remplies par des jeux d'ensemble variés, tels que saute-mouton, colin-maillard, jeux de barres, saut à la corde etc. et principalement les jeux de balle » (ARCHIVES MUNICIPALES DE METZ, 1932a). Cette description suggère le recours à des méthodes actives, conformément aux incitations émises par les textes officiels de 1923. Effectivement, beaucoup des instituteurs qui encadrent ces groupes d'élèves emploient des jeux qui peuvent être considérés comme traditionnels en France : balle cavalière, balle aux chasseurs, épervier, chat et souris, chat perché, petits paquets, jeu de barres ainsi que parfois football (ARCHIVES MUNICIPALES DE METZ, 1932f). L'instituteur Bonnet, qui dirige d'abord le groupe de garçons de l'école Paixhans puis à partir de juin celui de l'école de plein air de Bellecroix, fait pratiquer lui aussi à des jeux sportifs ainsi que le football et le handball (ARCHIVES MUNICIPALES DE METZ, 1932h). Pour ce sport, des réminiscences de l'influence du Reichsland sont perceptibles, puisque le handball ne se développe dans le reste de la France qu'après la seconde guerre mondiale (ATTALI, 2004). Par contre, certains instituteurs utilisent

4 Tous les lieux mentionnés représentent des quartiers de la ville de Metz ou de sa périphérie. Il en est de même pour tous les lieux mentionnés dans la suite de l'article. 
également des formes plus classiques de gymnastique, à l'instar de celles qui sont dispensées dans le cadre de l'enseignement obligatoire : le Règlement général d'éducation physique de 1925 prévoit en effet des assouplissements ou des exercices éducatifs pour développer la force et l'adresse (FRANCE, 1925). L'instituteur Michels, de l'école Saint-Martin déclare ainsi avoir, en plus de jeux, dispensé des séances de culture physique aux garçons de son groupe (ARCHIVES MUNICIPALES DE METZ, 1932g). Ce type d'exercices s'effectue en général de manière plus autoritaire, face à des élèves réduits à un statut de simples exécutants (FROISSART, 2012). Le programme des filles de l'école Saint-Maximin, dirigées par leur institutrice sœur Karst, est le suivant : " goûter 15 minutes; petite leçon de gymnastique 15 minutes ; jeux organisés avec balles et tambourins, cerceaux, cordes ; jeux libres ; rondes et marches » (ARCHIVES MUNICIPALES DE METZ, 1932b). Chez sœur Karst, les temps libres au cours desquels les élèves ne sont pas soumis à une surveillance stricte alternent avec une pédagogie plus dirigiste où les évolutions sont strictement contrôlées, comme la gymnastique, les jeux dirigés ou les rondes et marches.

Il existe aussi d'autres types d'animation spécifiques pour certains de ces groupes. Ainsi, l'instituteur Rohr emmène-t-il ses garçons de l'école Saint-Eucaire en promenade lors de 16 sorties consécutives (ARCHIVES MUNICIPALES DE METZ, 1932d), qui conduisent les élèves à effectuer entre 5 et 12 kilomètres de marche à pied, vers la route de Borny, la route de Sarrebrück, Saint-Julien, Vaux, Grigy. Le principe de ces promenades, développé lors des caravanes scolaires (HOIBIAN, 2016), consiste à organiser des sorties pédestres pour des élèves scolarisés sous la conduite d'enseignants volontaires. Ce procédé est éducatif et pluridisciplinaire, puisqu'il conduit l'instituteur à aborder des notions aussi diverses que les sciences naturelles, la géographie, l'histoire, le français... dans le cadre d'une école qui se veut ouverte sur le monde (DENIS, 2006). En l'occurrence, l'instituteur Mr Rohr exploite une possibilité offerte par les IO 1923 : le programme du cours supérieur présente, par rapport à celui du cours moyen, des nouveautés telles que « éducation des sens au cours des promenades scolaires » (IO 1923, p. 4). Lors des 6 dernières séances de l'année, les élèves de Mr Rohr ont droit à des bains à la piscine de plein air de la Natation messine (IO, 1923). De la même manière, l'instituteur Bonnet, lorsqu'il a en charge les 40 élèves de l'école de plein air de Bellecroix, les emmène à la piscine du stade de Bellecroix pour de l'ensoleillement et des bains (ARCHIVES MUNICIPALES DE METZ, 1932h). Cette école de plein air de Bellecroix ne semble pas être une école telle que celles créées en France depuis l'ouverture de la première du genre à Lyon en 1907 (VILARRET ; SAINT-MARTIN, 2004) qui fonctionnent durant toute l'année scolaire. Un courrier de la mairie indique en effet qu'une classe de plein air est ouverte 
au stade de Bellecroix, mais seulement à partir du 16 juin 1929 : une classe de filles est placée sous la responsabilité de l'institutrice A. Duroc de l'école de Saint-Julien lès Metz et une classe de garçons sous celle de A. Bonnet, instituteur à l'école de Paixhans (ARCHIVES MUNICIPALES DE METZ, 1930b). L'école de plein air de Bellecroix s'adresse, après accord de leurs parents, aux élèves détectés comme déficients par le médecin inspecteur scolaire : rachistisme, déficiences respiratoires, tuberculose, déviations vertébrales... peuvent être des raisons de leur inscription sur des listes d'enfants susceptibles de la fréquenter. Dans la mesure où les vacances scolaires d'été ne démarrent que le 15 juillet (GEBORD, 1999), ces élèves à besoin particulier bénéficient donc au moins d'un mois de cours au grand air au sein de cette structure située à côté du stade local.

On sait que les jeux de plein air ont fonctionné à Metz jusqu'en 1932. Cependant, les archives municipales ne comportent aucune trace qui témoignerait du prolongement de ces activités après cette date. Peut-être a-t-on assisté à leur suppression, suite à un avis défavorable du conseil municipal, face à la concurrence proposée par d'autres institutions d'encadrement de la jeunesse. Il est possible que les rapports envoyés en 1932 par les différents enseignants à la demande de la mairie n'aient pas convaincu les élus messins de la nécessité du maintien de ces jeux de plein air. Peut-être que, profitant de l'annonce du départ à la retraite du directeur François, les élus municipaux n'ont pas souhaité le remplacer (ARCHIVES MUNICIPALES DE METZ, 1932a), qu'ils n'ont pas voulu accéder aux revendications des enseignants qui demandaient un relèvement de leurs indemnités de 4 à 8 francs de l'heure (ARCHIVES MUNICIPALES DE METZ, 1932e). Une autre hypothèse résiderait dans le fait que la mairie désire dorénavant apporter son attention et donc ses subventions aux enfants les plus déficients. En effet, dans un courrier adressé à Mlle de Montlebert, médecin inspecteur des écoles, le conseil municipal l'enjoint de « soumettre une liste de 40 garçons et 40 petites filles au moins de neuf ans ayant besoin d'un séjour prolongé au grand air et choisis parmi les élèves des écoles élémentaires » (ARCHIVES MUNICIPALES DE METZ, 1930a). Il s'agit ici bien de favoriser le rétablissement corporel des élèves messins qui en ont le plus besoin, ce qui nécessite indéniablement des investissements financiers importants de la part de la municipalité messine et éventuellement l'abandon d'autres subventions. Sans que nous n'en ayons la preuve formelle, la municipalité messine a sans doute décidé de mettre un terme au fonctionnement des jeux de plein air en 1932. 


\section{Conclusion}

Ces divers exemples prouvent que la mise en place des jeux et exercices de plein air de la ville de Metz revêt des aspects protéiformes. Elle laisse des espaces de liberté aux enseignants dans le choix des activités proposées aux élèves. Elle octroie également davantage de liberté aux élèves dans le cadre des jeux et activités, même si à l'occasion, certains instituteurs et institutrices reviennent à des modalités plus cadrées, corrélatives de l'usage de pédagogies plus autoritaires, comme c'est l'usage dans l'enseignement obligatoire (GOMET, 2012). De plus, dans le cas du régime local particulier de l'entredeux-guerres, cette expérience des jeux de plein air revêt une dimension humaniste qui est débarrassée des contingences confessionnelles, ce qui est peu courant dans le milieu scolaire comme dans le milieu extra-scolaire mosellan. Et, bien qu'évoluant dans des espaces séparés, le fonctionnement est prévu autant pour les filles que pour les garçons. Ces élèves messins des écoles primaires bénéficient donc en général d'une éducation corporelle plus riche et plus variée que celles de leurs congénères qui ne fréquentent pas ces séances de plein air. Le cas des jeux de plein air proposés aux enfants volontaires des écoles messines entre 1919 et 1932 est un bon exemple de ce qui aurait pu constituer l'éducation physique obligatoire dans les écoles primaires après les IO 1923 : une discipline plus ouverte sur le plein air, le recours aux méthodes actives perceptibles à travers les jeux, une autonomie plus grande offerte aux élèves, qui contrasterait avec les séances d'éducation physique austères en réalité dispensées dans les écoles françaises.

En ce sens, il est heureux que des écoliers messins volontaires aient pu goûter, surtout dans une période où l'hygiénisme d'après-guerre est plus que jamais une priorité évidente, à un éveil au grand air dans un cadre périscolaire, même s'il ne concerne qu'une faible proportion d'entre eux. Selon Kieffer, 1994 (p. 315-336), on peut évaluer autour de 8000 le nombre d'élèves scolarisés dans le cycle primaire et en maternelle à Metz dans les années 1930. Seuls 300 à 500 d'entre eux, selon les années, ont bénéficié de ces jeux de plein air facultatifs. Ces dispositifs préfigurent et anticipent les mesures des arrêtés du 23 mars et 11 juillet 1938, où l'enseignement proprement dit se trouve allégé de 6 heures dont 3 sont consacrés à des exercices de sport et de plein air et réservées à des modes d`éducation plus libres, moins asservis aux méthodes qui s'imposent à l'intérieur de la classe (ARRÊTÉ, 1938a; 1938b). Finalement, sous ses aspects diversifiés, le plein air facultatif pour les écoliers de Metz a toujours ambitionné de donner à tous une éducation corporelle plus souple et qui poursuit plusieurs buts : former les corps des élèves, les rendre actifs, et leur inculquer des principes moraux. 


\section{RÉFERÉNCES}

ARCHIVES MUNICIPALES DE METZ. Courrier du directeur de l'école Saint-Maximin, Mr François, à la ville de Metz. Metz : 1 R 270, 20 juil. 1920a.

ARCHIVES MUNICIPALES DE METZ. Courrier du directeur de l'école Saint-Maximin, Mr François, à la ville de Metz. Metz : 1 R 270, 10 déc. 1920 b.

ARCHIVES MUNICIPALES DE METZ. Rapport du directeur de l'école Saint-Maximin, Mr François, à la ville de Metz. Metz : 1 R 270, 14 déc. 1922.

ARCHIVES MUNICIPALES DE METZ. Courrier du Directeur de l'Instruction publique de la Moselle au Préfet portant à sa connaissance la délibération (avis favorable) du conseil municipal de la ville de Metz sur le maintien des jeux scolaires. Metz: 1 R 270, 16 févr. 1923.

ARCHIVES MUNICIPALES DE METZ. Courrier du directeur François à la mairie de Metz. Metz : 1 R 270, 17 nov. 1924.

ARCHIVES MUNICIPALES DE METZ. Courrier du directeur de l'école Saint-Maximin, Mr François, à la ville de Metz. Metz : 1 R 270, 2 nov. 1925.

ARCHIVES MUNICIPALES DE METZ. Courrier de la ville de Metz à Mlle de Montlebert, médecin inspecteur des écoles. Metz : 1 R 270, avril 1930a (le jour n’est pas spécifié).

ARCHIVES MUNICIPALES DE METZ. Courrier de l'inspecteur d'académie de la Moselle à Monsieur le maire de Metz. Metz : 1 R 270, 29 mai 1930b.

ARCHIVES MUNICIPALES DE METZ. Bilan du directeur François adressé à la ville de Metz, Metz : 1 R 270, 4 févr. 1932a.

ARCHIVES MUNICIPALES DE METZ. Rapport annuel d'activité de sæur Karst, institutrice de l'école Saint-Maximin, à la mairie de Metz. Metz : 1 R 270, 7 févr. 1932b.

ARCHIVES MUNICIPALES DE METZ. Rapports d'activité annuelle demandés par la mairie de Metz: Mr Hubsch, école Saint-Vincent ; Mr Rohr, école Saint-Eucaire ; Mr Mathiotte, école Notre-Dame ; Mr Michels, école Saint-Martin ; Mr Bandines, école Saint-Maximin ; Mr Goury, école de Plantières-Queuleu ; Mr Reichert, école protestante de Queuleu; Mr Bonnet, école de Paixhans puis école de plein-air ; sœur Karst, école Saint-Maximin. Metz: 1 R 270, 22 févr. 1932c.

ARCHIVES MUNICIPALES DE METZ. Rapport annuel d'activité de l'instituteur Mr Rohr, école Saint-Eucaire. Metz : 1 R 270, 24 févr. 1932d.

ARCHIVES MUNICIPALES DE METZ. Courrier de François Hubsch, instituteur de l'école Saint-Vincent, à la ville de Metz. Metz : 1 R 270, 28 févr. 1932e.

ARCHIVES MUNICIPALES DE METZ. Rapport annuel d'activité de l'instituteur Mr Goury, école de Queuleu-Plantières. Metz : 1 R 270, 29 févr. 1932f. 
ARCHIVES MUNICIPALES DE METZ. Rapport annuel d'activité de l'instituteur Mr Michels, école Saint-Martin. Metz : 1 R 270, 21 mars 1932g.

ARCHIVES MUNICIPALES DE METZ. Rapport annuel d'activité de l'instituteur A. Bonnet, école de Paixhans. Metz : 1 R 270, 21 mars 1932h.

ARRÊTÉ du 23 mars 1938 modifiant l'horaire des Écoles primaires élémentaires. Paris : 23 mars 1938a.

ARRÊTÉ du 11 juillet 1938 modifiant les horaires des différents cours des écoles primaires élémentaires. Paris : 11 juil. 1938 b.

ARNAUD, Pierre. Le genre ou le sexe ? Sport féminin et changement social (XIXèmeXXème siècle). In : ARNAUD, Pierre ; TERRET, Thierry (org.). Histoire du sport féminin. Sport masculin-sport féminin : éducation et société. Paris : L'Harmattan, 1996. Tome 2, p. 147-183.

ATTALI, Michaël. Le hand-ball : l'invention d'un sport scolaire ? In : ATTALI, Michaël ; SAINT-MARTIN, Jean (org.). À l'École du sport. Épistémologie des savoirs corporels du XIXe siècle à nos jours. Louvain-la-neuve : De Boeck, 2014. p. 233-264.

BANCEL, Nicolas ; GAYMAN, Jean-Marc. Du guerrier à l'athlète. Éléments d'histoire des pratiques corporelles. Paris : PUF, 2002.

CHABOT, Charles. Le congrès d'hygiène scolaire et la Ligue des médecins et des familles. La Revue pédagogique, [s.l.], tome 43, p. 541-558, 1903.

CHARPIER, William. La Société des Gymnastes en Alsace (SGA) du milieu du $19^{\mathrm{e}}$ siècle à nos jours. In : TERRET, Thierry (org.). Histoire des sports. Paris : L'Harmattan, 1996. p. 11-37.

CRUBELIER, Maurice. L'enfance et la jeunesse dans la société française, 1800-1950. Paris : Armand Colin, 1979.

DELAPLACE, Jean-Michel. Culture et nature dans la « méthode naturelle » de Georges Hébert (1875-1957). In : SAINT-MARTIN, Jean ; TERRET, Thierry (org.). Le sport français dans l'entre-deux-guerres. Regards croisés sur les influences étrangères. Paris : L'Harmattan, 2000. p. 239-258.

DENIS, Daniel. Du dire au faire dans le dictionnaire de pédagogie : les arcanes d'une volonté républicaine de réformer l'éducation. Les sciences de l'éducation. Pour l'Ère nouvelle, Caen, v. 4, n. 39, p. 11-29, 2006.

DREIDEMY, Éric. La gymnastique à l'école pour germaniser l'Alsace-Lorraine (18701890). Staps, Paris, v. 2, n. 80, p. 7-22, 2008.

FRANCE. Ministère de la Guerre. Règlement général d'education physique: méthode française. Paris: Charles Lavauzelle, 1925-1930. t. 1-4.

FROISSART, Tony. La relation enseignants/élèves en EPS : processus éducatif subi ou agi ? In : OTTOGALLI-MAZZACAVALLO, Cécile ; LIOTARD, Philippe (org.). L'éducation du corps à l'école. Mouvements, normes et pédagogies, 1881-2011. Clermont-Ferrand : AFRAPS, 2012. p. 27-44. 
FUCHS, Julien. Concurrences et ententes au sein des mouvements de jeunesse. Le cas alsacien (1918-1960). Vingtième Siècle. Revue d'histoire, Paris, v. 3, n. 119, p. 113-126, 2013.

FUCHS, Julien ; STUMPP, Sébastien. Frontières politiques, frontières symboliques. La difficile implantation des organisations sportivo-touristiques allemandes en Alsace avant 1914. Revue d'histoire moderne \& contemporaine, Paris, v. 3, n. 60, p. 86-109, 2013.

GERBOD, Paul. Les rythmes scolaires en France : permanences, résistances et inflexions. Bibliothèque de l'école des chartes, Paris, Tome 157, livraison 2, p. 447-477, 1999.

GOMET, Doriane. Les pratiques des enseignants d'EPS dans le secondaire (1920-1980). Une facette peu connue de l'histoire de l'EPS. In : OTTOGALLI-MAZZACAVALLO, Cécile ; LIOTARD, Philippe (org.). L'éducation du corps à l'école. Mouvements, normes et pédagogies, 1881-2011. Clermont-Ferrand : AFRAPS, 2012. p. 75-88.

GUÉRIN, Christian. Le scoutisme français : une expérience pédagogique parallèle. Revue d'histoire moderne \& contemporaine, Paris, v. 1, n. 28, p. 118-131, 1981.

GAY-LESCOT, Jean Louis. Éducation physique et sport scolaires durant l'entre-deuxguerres (1919-1939) : des textes à la réalité. In : GLEYSE, Jacques (org.). L'éducation physique au XXème siècle. Approches historique et culturelle. Paris :Vigot, 1999. p. 59-68.

HOIBIAN, Olivier. L'œuvre des « caravanes scolaires » : un programme d'éducation globale à la périphérie de l'école républicaine (1874-1934). Revue française de pédagogie, Lyon, v. 2, n. 195, p. 25-36, 2016.

IO. Instruciones Oficiales. Plan d'études et programmes des écoles primaires élémentaires et instructions du 20 juin 1923. Paris, 20 juin 1923.

KIEFFER, Jean. L'enseignement primaire mosellan, de 1918 à 1939. Essai d'histoire sociale d'un particularisme scolaire. 1994. Thèse (Doctorat en Histoire) - Université de Lorraine, Metz, 1994.

LE BON, Frédéric. Une politique de l'enfance, du patronage au centre de loisirs. Éducation et sociétés, Paris, v. 1, n. 11, p. 135-152, 2003.

MANUEL d'exercices physiques et de jeux scolaires. Paris : Hachette, 1908.

OTTOGALLI-MAZZACAVALLO, Cécile ; LIOTARD, Philippe. L’apprentissage du genre en éducation physique. Devenir homme ou femme par l'exercice. In : OTTOGALLI-MAZZACAVALLO, Cécile ; LIOTARD, Philippe (org.). L'éducation du corps à l'école. Mouvements, normes et pédagogies, 1881-2011. Clermont-Ferrand : AFRAPS, 2012. p. 93-113.

PERNY, Pierre. L'arrivée des sports en Alsace. De l'éducation physique au culte de la compétition : un enjeu de société à la fin du XIXe siècle. Revue d'Alsace, Strasbourg, n. 140, p. 321-360, 2014.

PROST, Antoine. L'enseignement en France, 1800-1967. Paris : Armand Colin, 1968. 
QUIRIN-HÉMON, Isabelle. La germanisation par l'école en en Alsace-Moselle et en Poznanie : une politique coloniale? 2014. Thèse (Doctorat en Sciences de l'Homme et de la Société) - Université François Rabelais, Tours, 2014.

RAUCH, André. Le souci du corps. Paris : PUF, 1983.

ROSANVALLON, Pierre. L'État en France de 1789 à nos jours. Paris : Seuil, 1990. (Points Histoire).

ROTH, François. La Lorraine annexée, 1870-1918. Nancy : Presses universitaires de Nancy, 1976.

ROTH, François. Alsace-Lorraine. Histoire d'un « pays perdu ». De 1870 à nos jours. Paris : éditions Tallandier, 2016. (Texto).

SAINT-MARTIN, Jean. L'exemplarité des éducations physiques étrangères en France entre les deux guerres. Spirales, [s.l.], n. 13-14, p. 175-187, 1998.

SAINT-MARTIN, Jean. Les activités de nature à l'école : entre une approche vitaliste et un ancrage culturel. In : ATTALI, Michaël ; SAINT-MARTIN, Jean (org.). A l'école $d u$ sport. Épistémologie des savoirs corporels du XIXe siècle à nos jours. Louvain-laNeuve : De Boeck, 2014. p. 21-50.

SURREL, Yvonne. Plein Air : Éducation physique et activités dirigées. Paris : Éditions Bourrelier et Cie, 1947. (Carnets de l'éducation physique et des sports).

THIBAULT, Jacques. 1919-1929. La conjoncture des années d'après-guerre. In : ARNAUD, Pierre ; CLÉMENT, Jean-Paul ; HERR, Michel (org.). Éducation physique et sport en France. 1920-1980. Clermont-Ferrand : AFRAPS, 1995. p. 101-117.

VIGARELLO, Georges. Histoire de pratiques de santé. Le sain et le malsain depuis le Moyen-Âge. Paris : Seuil, 1999

VILLARET, Sylvain ; SAINT-MARTIN, Jean. Écoles de plein air et naturisme : une innovation en milieu scolaire (1887-1935). Movement \& Sport Sciences, Les Ulis, v. 1, n. 51, p. 11-28, 2004.

ZANCARINI-FOURNEL, Michelle. Genre des femmes et genre des corps. In : SAINTMARTIN, Jean ; TERRET, Thierry (org.). Sport et Genre. Apprentissage du genre et institutions éducatives. Paris : L'Harmattan, 2005. v. 3, p. 15-34.

Texte reçu le 04/10/2020.

Texte approuvé le 12/11/2020. 